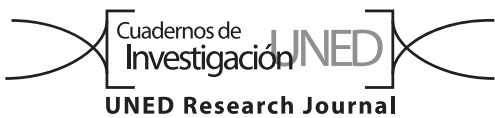

\title{
Uso de una aplicación como apoyo al aprendizaje de arreglos de la asignatura Lógica para computación de la Universidad Estatal a Distancia
}

\author{
Esteban Chanto Sánchez \& Carmen Andrés Jiménez
}

Universidad Estatal a Distancia (UNED), 474-2050 Mercedes de Montes de Oca, San José-Costa Rica; echanto@uned.ac.cr, candres@uned.ac.cr

Recibido 13-VI-2018 • Corregido 05-X-2018 • Aceptado 30-X-2018

\begin{abstract}
Use of an app as an arrangement learning support tool for Computation Logic subject of the Distance State University". Introduction: e-learning is growing around the world exponentially and locally adapted applications or "apps" are useful. Objective: to learn if low performing students in a course about unidimensional and multidimensional structures would be motivated to learn with the help of an app. Methods: we applied a survey to students of Computing Logic who used the app. Results: each student prefers didactical materials which can be used within their mobile dispositive and rated the app positively. Conclusions: the app must have video tutorials, solved exercises, readings and podcasts, as well as a section to set daily goals that can motive students. Furthermore, they want to be able to choose learning options that produce the same final result; and both students and teachers need to get involved with the app.
\end{abstract}

Key words: Information technology, distance education, computer app, mobile phone, educational computing.
RESUMEN: Introducción: El aprendizaje en línea crece en el mundo de una manera exponencial, el estudiantado cada vez necesita contar con el apoyo de dicha tecnología, por lo que el desarrollar una aplicación es un motivante para esta experiencia. Objetivo: determinar la anuencia del estudiantado a aprender mediante un material educativo que pueda ofrecerse en una aplicación fácilmente accesible. Metodología: indagamos sobre la posibilidad de utilizar un material educativo que se ofreciera mediante una aplicación fácilmente accesible mediante una encuesta. Resultados: cada estudiante prefiere materiales didácticos para su uso en los dispositivos móviles. El uso de una Aplicación es valorado positivamente como recurso para la comprensión de los temas planteados. Conclusiones: la aplicación debe ofrecer videos tutoriales, ejercicios resueltos, lecturas y podcasts; así como una sección de metas diarias que motiven la medición del aprendizaje. Recomendamos ofrecer diferentes opciones para aprender y elementos didácticos y técnicos dentro de la aplicación. Además, la comunidad estudiantil y el personal docente deben involucrarse en la comprensión del contenido de la aplicación.

Palabras clave: tecnología de la información, educación a distancia, aplicación informática, teléfono móvil, informática educativa.
El aprendizaje en línea está creciendo en el mundo de una manera exponencial, como nunca antes visto y el estudiantado está anuente a utilizar todo tipo de aplicaciones, software, plataformas y demás opciones que la tecnología le pueda ofrecer. La Universidad Estatal a Distancia de Costa Rica no es la excepción y el avance que se ha logrado es tangible y ha generado resultados positivos tanto en la calidad de las asignaturas de la oferta académica como en el rendimiento de la población estudiantil. En el Plan de desarrollo académico de la UNED (2012), se destacan las políticas para la innovación e integración de las tecnologías digitales que se deben utilizar en los procesos de enseñanza y aprendizaje, así como la relevancia que implica la inclusión de la población estudiantil en dichos procesos.
El desarrollar una aplicación para un sector de la población estudiantil relacionado con la tecnología, es con mayor razón un motivante para este estudio. Para García, Ruiz y Domínguez (2007), el aprendizaje móvil permite la integración del e-learning con los dispositivos móviles y así favorecer el aprendizaje ubicuo; lo anterior evidencia la importancia de implementarlo en la UNED como una muestra de mejoramiento continuo y de brindar mayores posibilidades de acceso a la población para el estudio de sus asignaturas.

De estas opiniones se infiere que los educandos aprovechan su tiempo para acceder a la plataforma y realizar actividades académicas de las asignaturas que matriculan y lo requieren de las universidades cada vez más como un agente motivador. Según Pechenkina, 
Laurence, Oates, Eldridge y Hunter (2017), aunque aún se requiere más investigación en el tema, el uso de aplicaciones móviles ha logrado que el rendimiento de los estudiantes mejore y ha mantenido a dicha población interesada en sus estudios, lo que se convierte en una ventaja para el profesorado.

Por otro lado, el aprendizaje móvil es de una naturaleza particular, que no sustituye a la metodología tradicional de enseñanza por ser móvil, personalizado y cooperativo, entre otras características, tal y como lo señala la UNESCO (s.f.). Tal hecho resalta características esenciales como la portabilidad e interactividad, y que el aprendizaje móvil posee elementos del e-learning, pero son distintos en su desarrollo y medios de difusión. Por lo tanto, es necesario considerar las tecnologías móviles para el desarrollo de la propuesta y la penetración de estos aparatos en el mercado nacional.

Según Castaño y Cabero (2013) la UNESCO realiza una fuerte apuesta a la tecnología móvil, ya que permite llegar a zonas lejanas donde la manera en la que se puede ingresar a la información es mediante tecnologías móviles.

Tal razonamiento tanto de cada autor como de la UNESCO permite a la educación analizar e incluir las tecnologías móviles con estrategias inclusivas que coadyuven en los procesos formativos de las personas. A este respecto, Salas (2016) señala que al ocurrir un cambio radical en las tecnologías de la información, la manera en la que se transmite el conocimiento también se modificará.

La anterior es una evolución en la población estudiantil que se está viviendo día a día en las universidades que utilizan este tipo de opciones para transmitir conocimiento: ya no desde un aula, ya no desde el sistema presencial; sino desde cualquier lugar, en cualquier momento y como a la persona le sea más beneficioso, lo cual también trae consecuencias muy favorables en el campo socioeconómico afectivo de las personas. Figueras-Maz, Ferrez y Mateus (2018), indican que la tecnología está al servicio de la innovación, pero es importante situar la pedagogía delante de cualquier componente tecnológico.

Una vez establecidos los pilares bajo los cuales este estudio se sustente, se puede señalar que el problema planteado fue: ¿Puede fortalecerse la comprensión de las estructuras unidimensionales y multidimensionales, para la resolución de problemas algorítmicos en la asignatura de Lógica para computación? De igual manera, a partir del problema planteado, se estableció el objetivo de esta experiencia, el cual es determinar la anuencia del estudiantado de la asignatura "Lógica para computación" del Diplomado en informática de la Universidad Estatal a Distancia a aprender mediante un material educativo que pueda ofrecerse en una aplicación fácilmente accesible.

\section{MATERIALES Y MÉTODOS}

Cuando se realizó el diagnóstico en la población estudiantil se hizo con dos propósitos fundamentales: el primero, conocer la tenencia de los dispositivos móviles en los estudiantes que matriculan la asignatura "Lógica para computación"; y el segundo, conocer la preferencia de material y medio, para el apoyo en el proceso de enseñanza, para los temas de arreglos unidimensionales y multidimensionales.

Procedimiento de resolución de problemas: en el área de sistemas de información, específicamente en el análisis algorítmico de la resolución de problemas, existe la metodología relacionada con resolución de problemas computacionales. Esta consta de cinco fases que garantizan el análisis de una situación y resolución de un problema en el área; por esta razón, se ha elegido dicha metodología para la presentación de los algoritmos, su análisis y solución para este estudio. Las fases se explican a continuación.

1. Identificación del problema: de este análisis depende el desarrollo de la solución. El problema se acota la información de entrada y salida para determinar los datos requeridos y plantear la solución.

2. Planteamiento de alternativas de solución: posterior a la definición del problema, se continúa con el análisis de las opciones de solución. Generalmente los problemas se pueden resolver de diferentes formas. Se pueden plantear varias alternativas de solución; no obstante, muchas alternativas pueden dificultar la escogencia de la selección más adecuada.

3. Elección de una alternativa: posterior a la gama de opciones para solucionar el problema, se procede a elegir la más eficiente. Este punto es importante porque cambiar en el camino la solución elegida resulta muy costoso, ya que implica empezar desde cero con una nueva opción.

4. Desarrollo de la solución: después de elegir la opción más eficiente, se desarrolla la solución del problema, esto implica la elección de operaciones, procesos; entre otros.

5. Evaluación de la solución: al finalizar el desarrollo de la solución, se procede a evaluarla. Esto es conocido como control de la calidad, en donde se comprueba que los resultados obtenidos fueron correctos. En esta fase se deben mejorar los procedimientos y hacerlos más eficientes, para que se invierta la menor cantidad de recursos posibles (Palmero, 2011). 
Tipo de investigación: la investigación realizada es de tipo cuantitativa, sobre la cual, Hernández, Fernández y Baptista (2006) indican que "el enfoque cuantitativo pretende intencionalmente "acotar" la información (medir con precisión las variables de estudio....)" (p.10).

Dicho enfoque busca ser objetivo, aplica la lógica deductiva de lo general a lo particular y el investigador es neutral e imparcial, donde intenta asegurar procedimientos rigurosos y objetivos de recolección y análisis de datos (Hernández, Fernández, \& Baptista, 2006).

El diseño del estudio es no experimental de tipo transeccional descriptivo. De acuerdo con Hernández, Fernández y Baptista (2006) "Ios diseños transeccionales realizan observaciones en un momento único en el tiempo (...) cuando recolectan datos sobre cada una de las categorías, conceptos, variables, contextos, comunidades o fenómenos, y reportan lo que arrojan esos datos son descriptivos (...)" (p. 165).

Población en estudio: la población comprende a todo el estudiantado que matriculó dos asignaturas: Lógica para computación (3071) y Matemática para computación II (3069), en los bloques A y B del diplomado de Ingeniería Informática durante el primer cuatrimestre del 2016. Ambas asignaturas son propias de la especialidad de la carrera y se omiten aquellas relacionadas con Estudios Generales. La población total corresponde a $1157(\mathrm{~N}=1157)$.

Se tiene una muestra probabilística y para elaborar el cálculo de su tamaño, se empleó una calculadora en línea (Create Research Systems, 2006), de manera que fuera representativa de la población. Se eligió un nivel de confianza del $95 \%$, con un intervalo de confianza del 5\% y una población de 1157 estudiantes. Con dichos datos, la muestra representativa fue de 289 .

Para buscar el nivel de confianza, se emplearon los siguientes valores: $95 \%$ nivel de confianza, con un tamaño de muestra de 440 (que fueron las respuestas obtenidas en el cuestionario), con la población de 1157 estudiantes del I cuatrimestre 2016 y con un porcentaje del $47 \%$, se determinó un intervalo de confianza de 3,67. La respuesta final estuvo constituida por el estudiantado de la muestra que voluntariamente respondió el cuestionario auto administrado. Se obtuvo un total de 440 respuestas, por lo que los resultados pueden considerarse significativos para la población elegida.

Elaboración de cuestionario: el instrumento para recopilar la información consistió en un cuestionario auto administrado, constituido por 19 ítems, de los cuales, 12 fueron de selección única, seis de selección múltiple y uno, de pregunta abierta. Los ítems se elaboraron para recolectar información sobre:

- Datos demográficos: sexo, edad, provincia de residencia.

- Matrícula: asignaturas matriculadas.

- Dispositivos: tenencia, sistema operativo, proveedor, tipo de contrato y frecuencia de uso.

- Recursos y materiales: recursos para el aprendizaje, preferencia, elementos a considerar y uso.

- Aplicaciones: uso, instalación, tipos de aplicaciones empleadas, utilidad para el aprendizaje, frecuencia de uso, aplicaciones significativas.

El cuestionario fue validado por especialistas en educación e ingeniería informática; ambos grupos tienen conocimiento en el uso de materiales didácticos.

Para la construcción del cuestionario, se realizó una tabla de tránsito de la variable al ítem. Después de recopilar la información brindada por el estudiantado, se realiza un análisis descriptivo de los datos recopilados por medio del programa surveymonkey.net, que permite la extracción de los datos y algunas relaciones de información entre las variables. El análisis se hizo debido a las variables definidas (ver Apéndice Digital 1 para cuestionario).

Ética, conflicto de intereses y declaración de financiamiento: los autores declaran haber cumplido con todos los requisitos éticos y legales pertinentes, tanto durante el estudio como en el manuscrito; que no hay conflictos de interés de ningún tipo, y que todas las fuentes financieras se detallan plena y claramente en la sección de agradecimientos. Asimismo, están de acuerdo con la versión editada final del documento. El respectivo documento legal firmado se encuentra en los archivos de la revista.

\section{RESULTADOS}

A continuación, se muestran los resultados de un diagnóstico realizado a estudiantes de la asignatura de Lógica para computación de la UNED Costa Rica en el primer cuatrimestre del 2016 y su opinión sobre la preferencia de materiales en dicha asignatura para la comprensión de las estructuras uni y multidimensionales.

El resultado del diagnóstico realizado es que los estudiantes prefirieron el desarrollo de una Aplicación, con 
ejercicios resueltos y video-tutorías. Es importante resaltar que, la creación de cualquier propuesta educativa responde a elementos didácticos referentes al abordaje de contenidos, metáforas del entorno, ejercicios de evaluación, entre otros; y a elementos técnicos que engloban la navegación, accesibilidad, usabilidad, propuesta gráfica y programa donde se desarrolla. Por ello, se abordan tanto los elementos didácticos como técnicos en la elaboración del proyecto.

Características del estudiantado entrevistado: se estudiaron diferentes características del estudiantado para tener una noción de este. Se encontró que el 69,57\% de los encuestados corresponden a hombres y el 30,43\% a mujeres. Respecto a la distribución del estudiantado por rango de edad, los educandos se ubican mayoritariamente en un rango de población adulta joven comprendidos entre los 18 y 41 años con un 97,02\% (Cuadro 1).

Sobre la provincia de residencia de los educandos, el $47,37 \%$ de las personas que contestaron este cuestionario, pertenecen a la provincia de San José. Las provincias donde se encuentran ubicados el $89,48 \%$ del estudiantado, pertenecen al Gran Área Metropolitana [GAM].

Dispositivos y características: con respecto a la tenencia de dispositivos entre las personas de primer ingreso a la carrera del Diplomado de Informática se encontró que el dispositivo con que más cuentan los encuestados es de teléfonos inteligentes (con un 90,59\%), seguido de un $82,67 \%$ de personas con computadoras portátiles. En menor porcentaje de computadoras de escritorio con un $48,51 \%$ y las tabletas con un $32,18 \%$ (Fig. 1 ).
Sobre el sistema operativo de los dispositivos, se obtuvo que Android tuvo un $76,24 \%$ de incidencia, seguido por un $12,38 \%$ de iOS y un 7,92\% de Windows 10 (Cuadro 1).

CUADRO 1

Sistemas Operativos de los dispositivos

\begin{tabular}{|c|c|}
\hline Opción & Porcentaje (\%) \\
\hline Android & 76,24 \\
\hline IOS (Aplicaciónle) & 12,38 \\
\hline Windows 10 & 7,92 \\
\hline Windows Phone & 1,98 \\
\hline Symbian & 0,50 \\
\hline No lo sé & 0,50 \\
\hline Blackberry & 0,50 \\
\hline Palm OS & 0,00 \\
\hline
\end{tabular}

Sobre la cantidad de horas al día que el estudiantado invierte en el uso del teléfono móvil para navegar por Internet, el 35,64\% de los encuestados respondieron que usan el dispositivo más de 8 horas, seguido de un $27,23 \%$ que lo emplean de 3 a 4 horas y un $18,81 \%$ de 5 a 7 horas de uso.

Recursos para el aprendizaje: se consultó la preferencia del estudiantado sobre el tipo de recurso educativo que la UNED podría desarrollar para apoyar al estudiante en su proceso de aprendizaje. Se encontró que el tipo de recurso educativo de preferencia es el Aplicación para dispositivos móviles con un 81,19\%

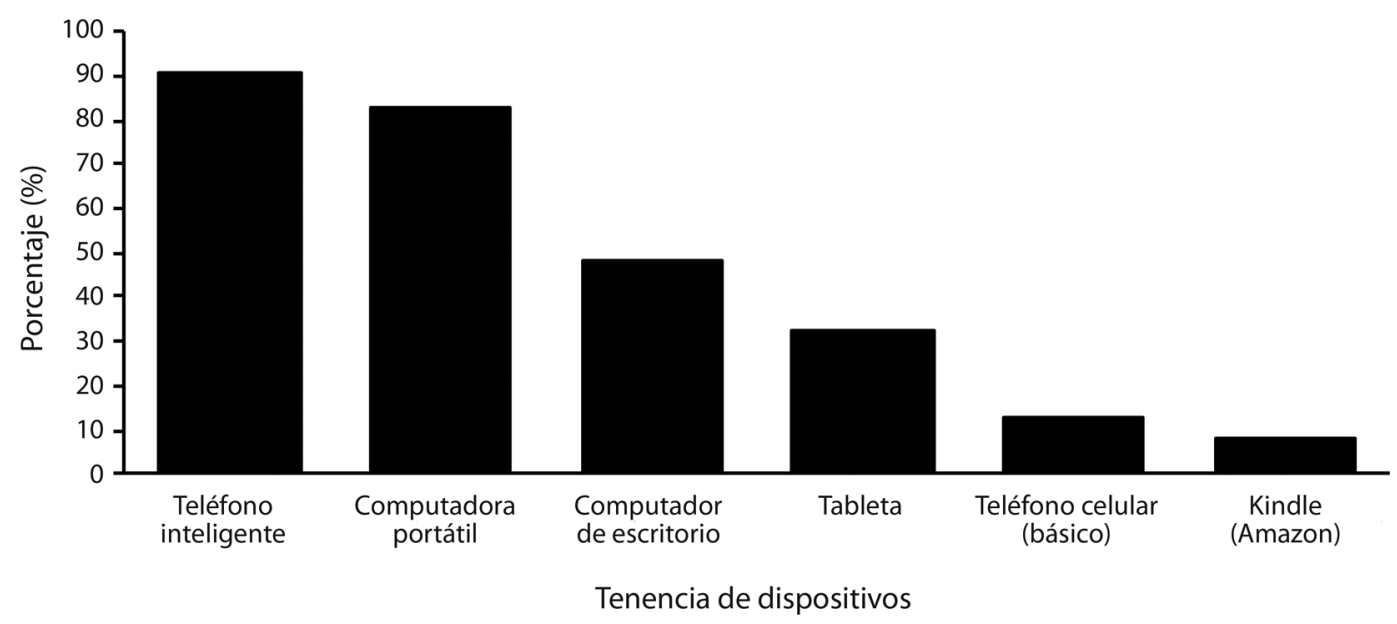

Fig. 1. Tenencia de dispositivos móviles entre personas de primer ingreso a la carrera del Diplomado de Informática. 


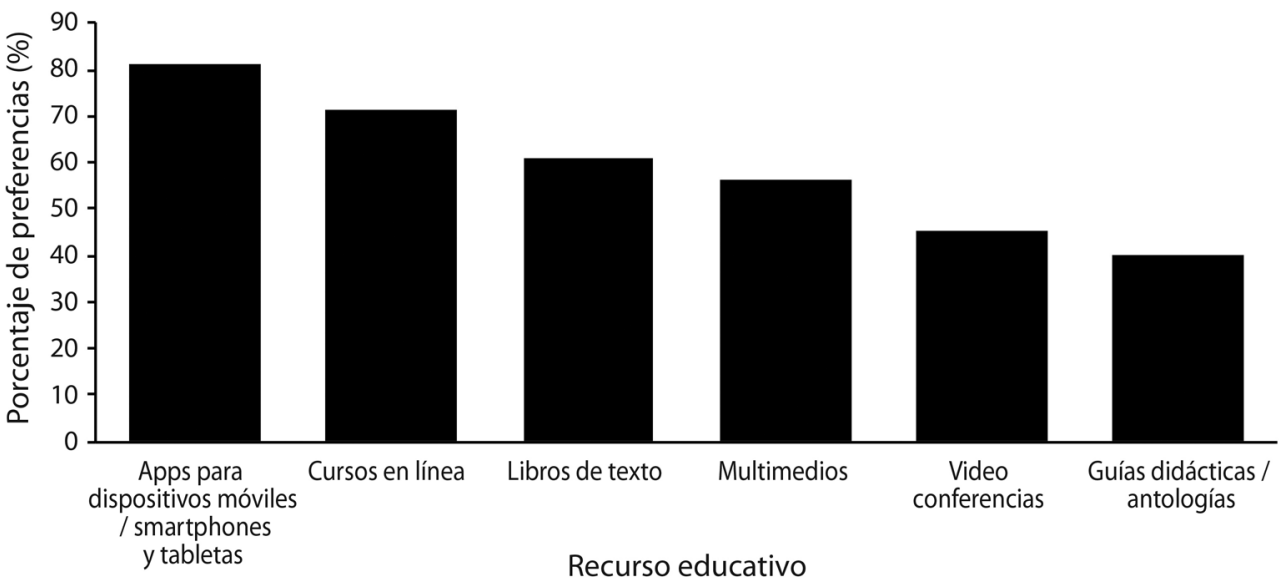

Fig. 2. Preferencia de recursos educativos para el aprendizaje.

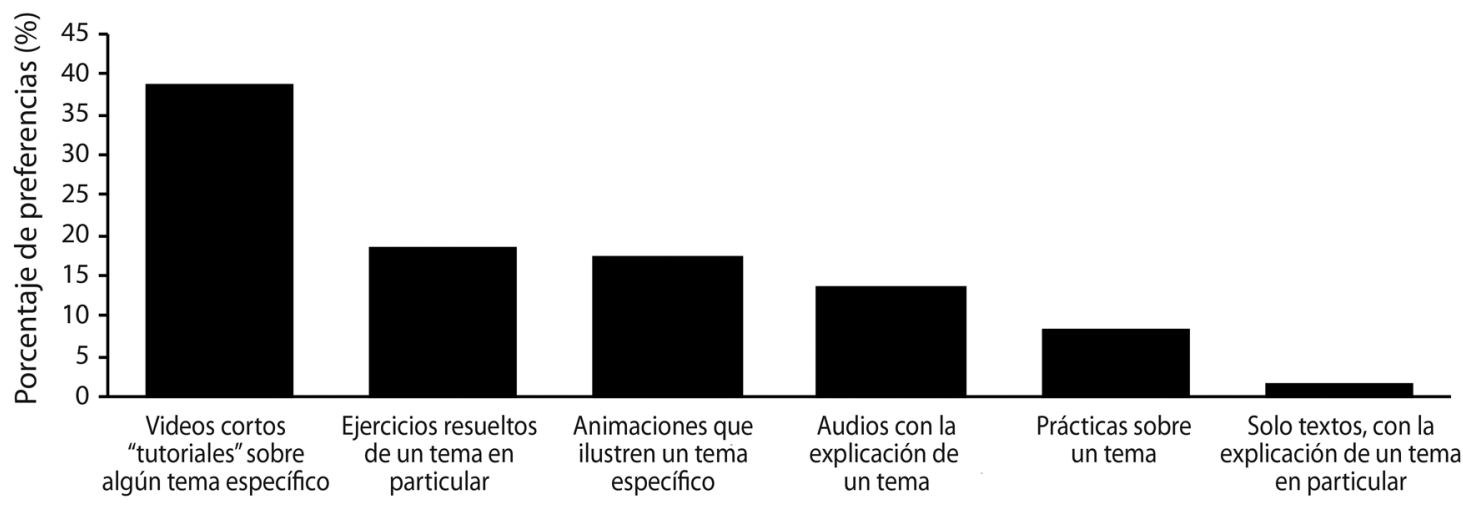

Elementos para el aprendizaje

Fig. 3. Preferencia de elementos para el aprendizaje de Lógica para computación.

seguido de los cursos en línea con un 71,29\% y luego los libros de texto con un 60,89\% (Fig. 2).

A las personas que eligieron haber matriculado la asignatura de Lógica para computación durante el I cuatrimestre 2016, se les consultó cómo les gustaría estudiar los temas de la asignatura, esto con la finalidad de conocer los elementos que consideran que favorecen el aprendizaje. Los elementos más importantes que los encuestados indicaron como relevantes, un 38,81\% indicó que desean contar con videotutoriales, seguido de un $18,52 \%$ de encuestados que prefieren contar con diferentes ejercicios resueltos de un tema particular (Fig. 3).

Al igual que la pregunta anterior, al estudiantado que indicó haber matriculado la asignatura de Lógica para computación se le consultó los aspectos que le gustaría que tenga una Aplicación para la asignatura 3071 (Fig. 4).

Finalmente, a estos estudiantes matriculados en la asignatura 3071 , se les consultó si estarían dispuestos a utilizar una aplicación educativa diseñada para dispositivos móviles que sea de apoyo al proceso de aprendizaje, el $96,50 \%$ respondieron de forma afirmativa, frente a un $3,50 \%$ que indicaron que no la usarían.

Uso de aplicación: para conocer la experiencia en la descarga o utilización de las aplicaciones para dispositivos móviles que ha tenido el estudiantado, se les consultó si han empleado estas en su aparato. El 97,01\% sí tienen experiencia, frente a un 2,99\% que indicaron que no.

También se consultaron los tipos de aplicaciones que el estudiantado han usado o instalado en su dispositivo. Sobre ello, se observa que el $84,65 \%$ de los encuestados usan aplicaciones educativas, seguido de un $83,66 \%$ usan aplicaciones de comunicación y con un $80,20 \%$ del estudiantado encuestado usan aplicaciones relacionadas con las redes sociales (Fig. 5). 


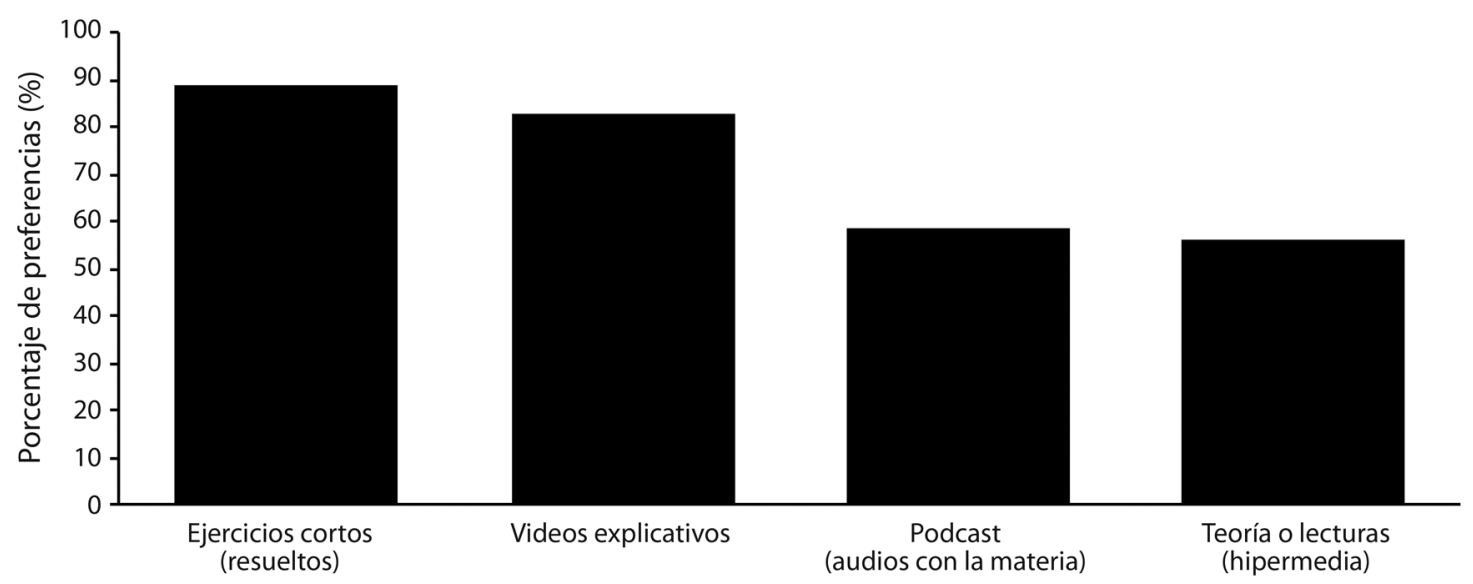

Elementos para una App educativa

Fig. 4. Elementos para una aplicación educativa de la asignatura 3071.

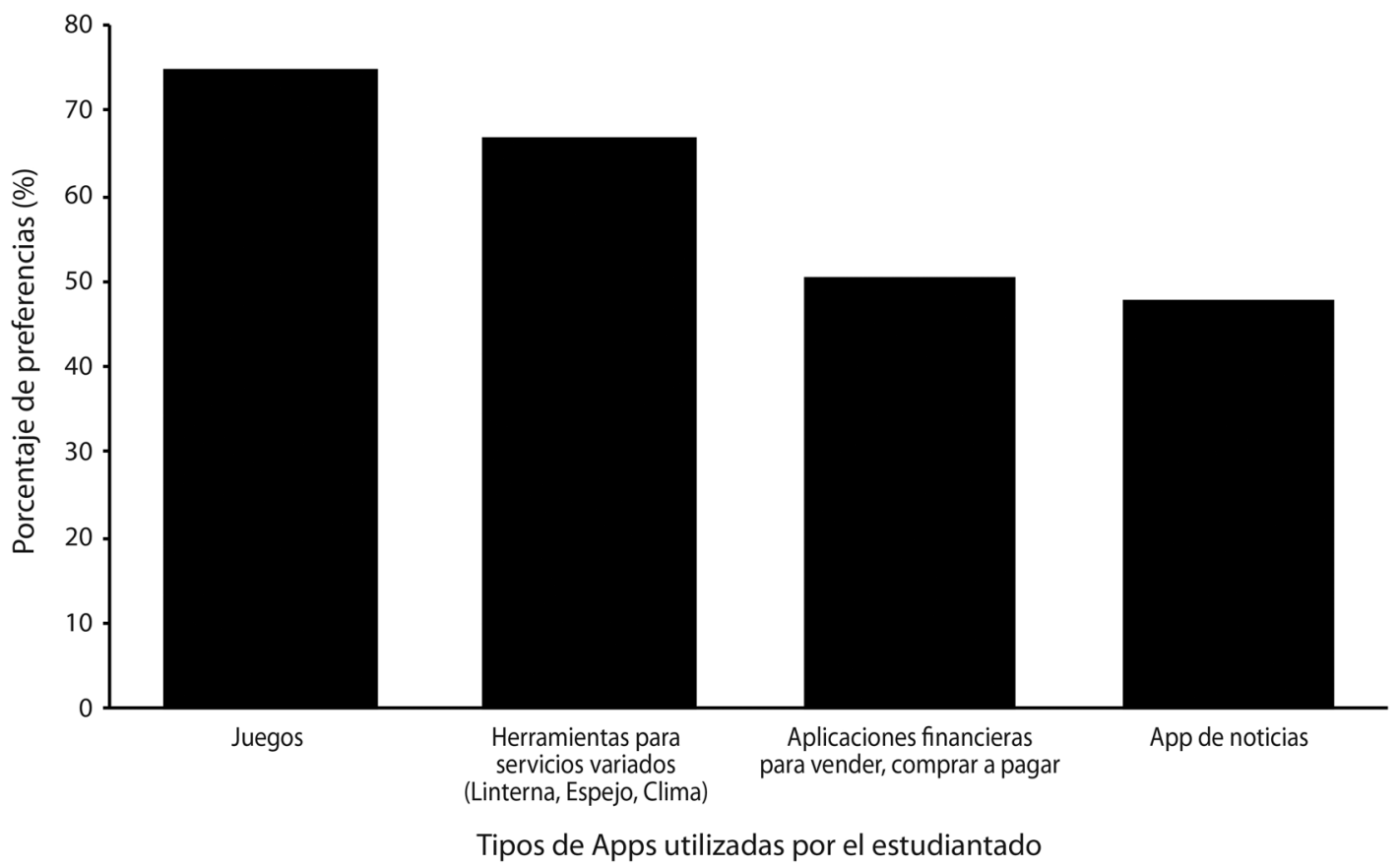

Fig. 5. Tipos de aplicaciones utilizados por el estudiantado.

Referente al uso del dispositivo móvil, el 85,15\% del estudiantado utilizan su dispositivo para realizar y contestar llamadas telefónicas, seguido con un $84,65 \%$ para navegar por Internet, y ese mismo porcentaje para chatear (Fig. 6).

También se recopiló la opinión relacionada a la percepción que pueden tener los dispositivos móviles como herramientas para facilitar el aprendizaje (Fig. 7).
Un elemento consultado en la población encuestada es si utilizarían una Aplicación educativa para apoyar el proceso de aprendizaje (Fig. 8).

Finalmente, se consultó al estudiante sobre las Aplicaciones que conoce cuál le dejó una enseñanza significativa para la vida. Sobre las respuestas obtenidas, el Cuadro 2 muestra que la opción de mayor incidencia es Aplicación de variables de la asignatura de Lógica para computación con un $56,60 \%$. 


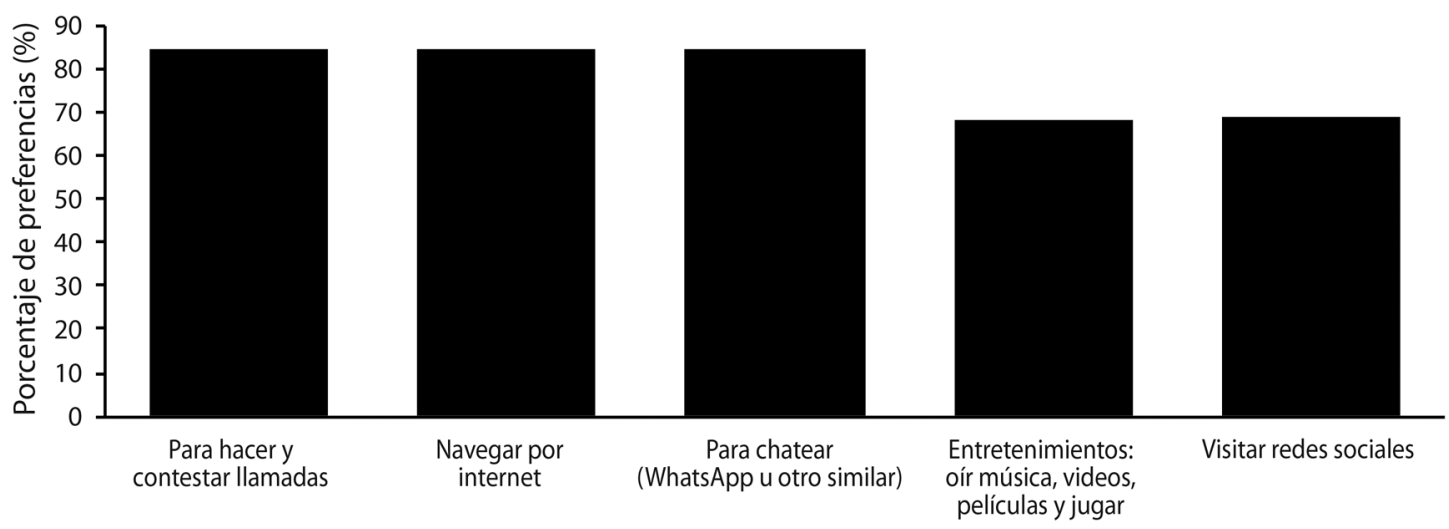

Uso del dispositivo móvil por parte del estudiantado

Fig. 6. Uso del dispositivo móvil por parte del estudiantado.

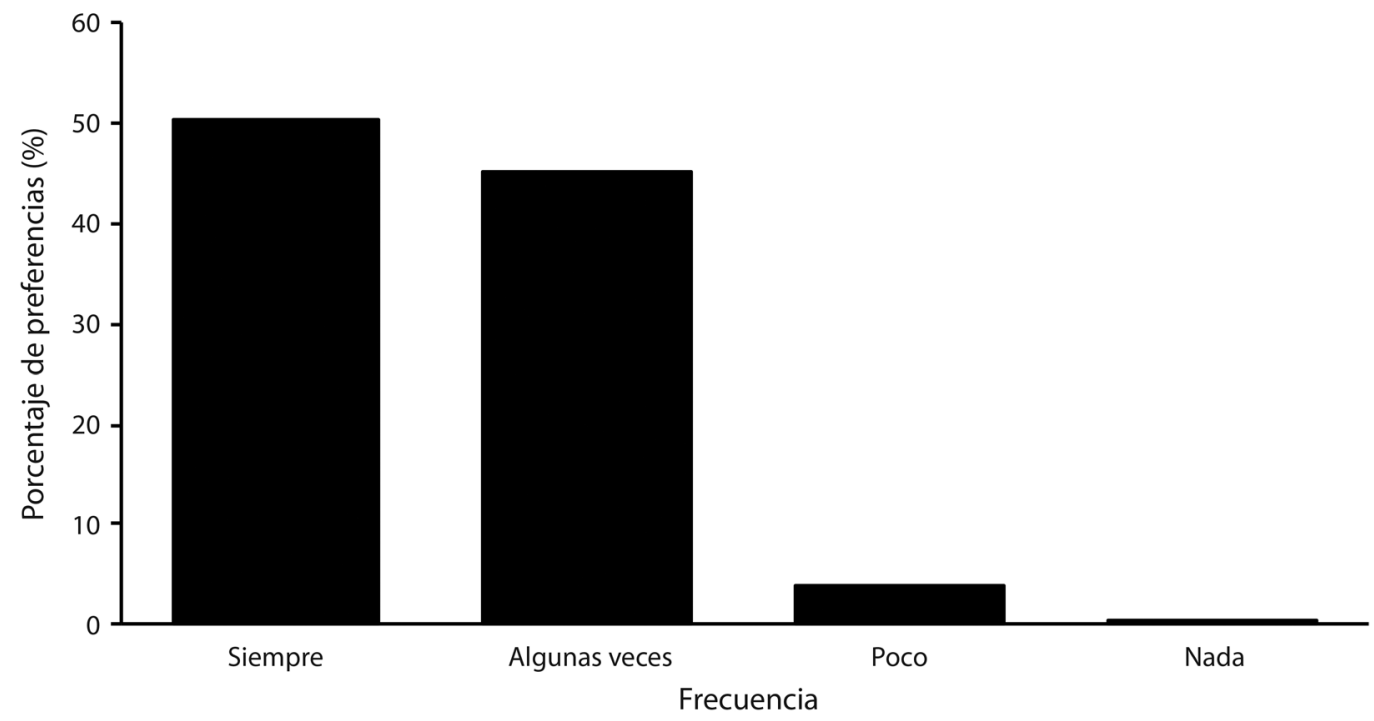

Fig. 7. Percepción de la facilitad del aprendizaje a través de dispositivos móviles.

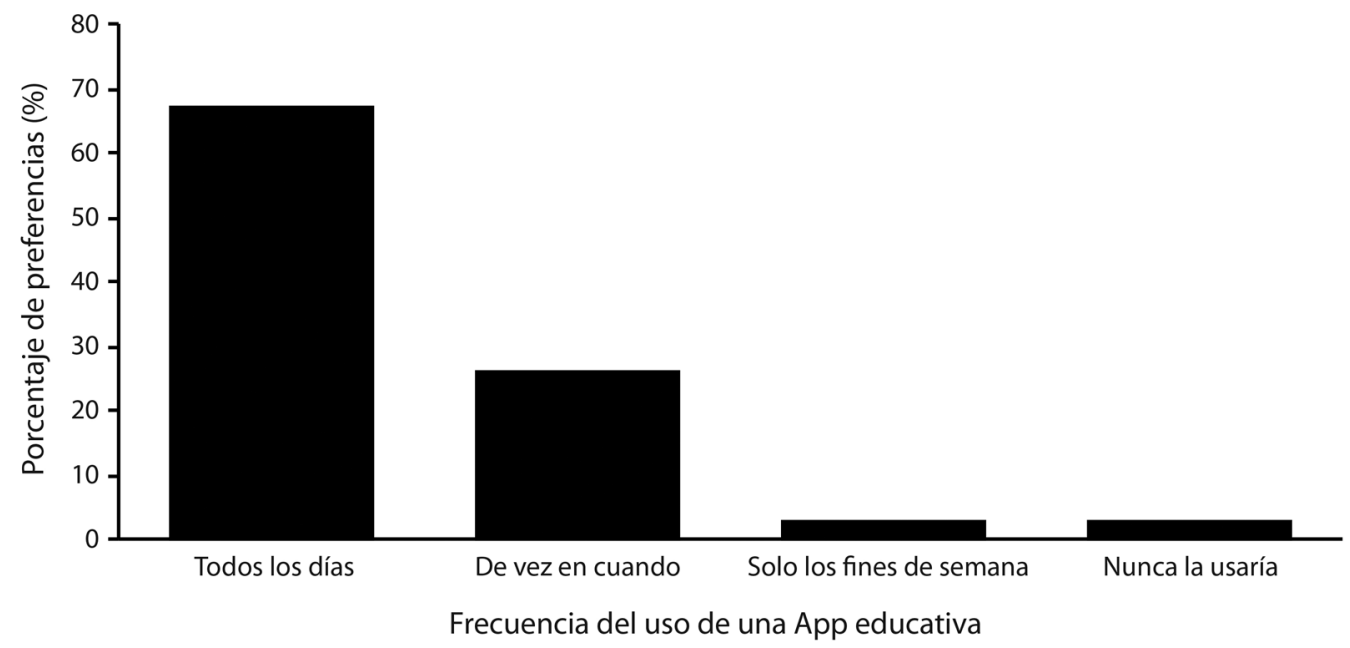

Fig. 8. Utilización de una aplicación educativa. 
CUADRO 2

Aplicaciones significativas para el estudiantado

\begin{tabular}{|c|c|}
\hline Opción & Porcentaje (\%) \\
\hline $\begin{array}{l}\text { Aplicación que explica que son las variables del } \\
\text { curso de lógica de computación }\end{array}$ & 56,60 \\
\hline Otras Aplicaciones (especifique) & 30,19 \\
\hline Prueba teórica del examen de conducir COSEVI & 6,60 \\
\hline $\begin{array}{l}\text { Estacionescr permite ubicar por GPS todas las } \\
\text { estaciones de servicio del país }\end{array}$ & 2,83 \\
\hline $\begin{array}{l}\text { Aplicación para la feria vocacional de la Universidad } \\
\text { de Costa Rica }\end{array}$ & 2,83 \\
\hline $\begin{array}{l}\text { Transportecr una aplicación para consultar las tarifas } \\
\text { del transporte público }\end{array}$ & 0,94 \\
\hline
\end{tabular}

Sobre las otras Aplicaciones que indicaron el estudiantado con una incidencia del 30,19\% se citaron las indicadas en el Cuadro 3, de dichas Aplicaciones, se observa que las categorizadas como educativas, tienen una gran variedad en las áreas de preferencia.

CUADRO 3

Otras Aplicaciones empleadas por el estudiantado

\begin{tabular}{|c|c|c|}
\hline Categoría & Especialidad / Área & Aplicación \\
\hline \multirow[t]{5}{*}{$\begin{array}{l}\text { Aplicaciones } \\
\text { educativas }\end{array}$} & Idiomas & $\begin{array}{l}\text { Inglés plus } \\
\text { vídeos educativos inglés } \\
\text { Traductor inglés a español }\end{array}$ \\
\hline & Ciencias & $\begin{array}{l}\text { Geografía } \\
\text { Química } \\
\text { Aves de CR } \\
\text { Soluciones Químicas }\end{array}$ \\
\hline & Matemáticas & $\begin{array}{l}\text { Matemática plus } \\
\text { Juegos de matemática Zeus } \\
\text { Calculadora de ecuaciones }\end{array}$ \\
\hline & Apoyo a cursos & Google classroom \\
\hline & Diccionarios & Diccionario de costarriqueñismos \\
\hline \multirow[t]{5}{*}{$\begin{array}{l}\text { Otras } \\
\text { Aplicaciones }\end{array}$} & $\begin{array}{l}\text { Lecturas y } \\
\text { prácticas }\end{array}$ & La Biblia \\
\hline & Prácticas & Examen teórico manejo \\
\hline & $\begin{array}{l}\text { Aplicaciones de } \\
\text { entretenimiento }\end{array}$ & $\begin{array}{l}\text { Puzzle } \\
\text { Teletica interactiva } \\
\text { Lumosity Brain Training } \\
\text { Radio Costa Rica }\end{array}$ \\
\hline & Viajes & Despegar.com \\
\hline & Estilo de vida & $\begin{array}{l}\text { NutriSalud NS } \\
\text { Recetas de tía Florita }\end{array}$ \\
\hline
\end{tabular}

\section{DISCUSIÓN}

El hecho de que la mayor parte del estudiantado resida en la GAM se complementa con la información de la Superintendencia de Telecomunicaciones (SUTEL, 2018) donde el mapa de cobertura indica para los tres operadores de telefonía e Internet, que la GAM es el área con mayor acceso a servicios. Esto sugiere que la cobertura y la estabilidad de la señal serán altas, dando más ventajas y posibilidades para una mayor comprensión por parte de cada discente al utilizar la aplicación.

Dado que se muestra una opinión positiva hacia el uso de una aplicación móvil para aprender, va aunado al hecho de que todo lo que represente la virtualización de la educación y la disminución de la presencialidad, es beneficioso para la población estudiantil y cada vez más solicitado por esta. En un estudio realizado por Vázquez y Sevillano (2018) se comprueba esta teoría y reafirma que el estudiantado está cada vez más anuente a estudiar a través de la virtualidad. De igual manera, otro estudio realizado por Arain, Hussain, Rizvi, y Vighio (2017), encontró este mismo resultado entre la población estudiantil entrevistada, además de haber determinado una diferencia estadísticamente significativa entre el rendimiento del estudiantado que utilizó aplicaciones móviles y los que estudiaron bajo una modalidad tradicional.

El razonamiento anterior se fundamenta con los resultados obtenidos, que se muestran en la Fig. 1, respecto a que gran parte del estudiantado tiene teléfonos inteligentes y el hecho de que son dispositivos de uso diario y muy frecuente; todo lo cual, hace factible esta manera de impartir la asignatura.

Otro de los resultados encontrados se relaciona con el hecho de que aparte del uso habitual de llamadas, tanto los hombres como mujeres emplean el dispositivo para chatear y navegar por Internet y en menor grado lo emplean para el entretenimiento y redes sociales, además que tener una percepción positiva sobre el uso de estos dispositivos como apoyo al proceso de aprendizaje y estar dispuestos a utilizar una aplicación móvil en la asignatura en estudio 3071, podría sugerir que el celular es un medio mediante el cual podrían también aprender. De acuerdo con un estudio realizado por De Witt y Gloerfeld (2017), en el que se estudió el aprendizaje móvil, se encontró que las universidades utilizan este tipo de enseñanza para prolongar las lecciones presenciales, lo cual genera una nueva manera de aprender.

Por otro lado, el hecho de que un porcentaje muy alto (68\%) de la población encuestada hace uso de las aplicaciones de comunicación y aplicaciones académicas y muy pocos de aplicaciones relacionadas con noticias, sugiere que el estudiantado estaría abierto al uso de una Aplicación educativa. Un estudio realizado en Malasia por Ayub, Zaini, Luan y Jaafar (2017), encontró que el uso de tecnologías emergentes en la educación superior posee en sí mismo un componente motivacional que 
genera en el estudiantado resultados muy positivos en su rendimiento y en innovación personal, así como en su preparación para la siguiente clase.

Asimismo, como un porcentaje alto de la población utiliza líneas pospago y por ocho horas al día y; por el contrario, el resto de la población tiene línea prepago y la utiliza menos de una hora al día; es esencial tomar en cuenta este detalle en el diseño de la aplicación móvil, de manera que la población con menos acceso a internet no vaya a ser afectada. Sin embargo, un estudio realizado por Pechenkina (2017), indica que una de las expectativas del estudiantado es tener este tipo de aplicaciones dentro del material a estudiar en sus asignaturas, por lo que la universidad está en obligación de ir de la mano con estas tecnologías emergentes y satisfacer las necesidades, sin dejar de lado las limitaciones que cada población pueda tener.

Seguidamente, de los resultados obtenidos respecto a los recursos para el aprendizaje que se podrían incluir en la aplicación, se puede inferir que esta debe de manera indiscutible ofrecer videotutoriales, ejercicios resueltos, lecturas y podcasts. Lo anterior aunado al hecho de que la mayoría de las personas respondieron que creen que el aprendizaje se vuelve fácil a través de dispositivos móviles, además de que estarían dispuestos a utilizarla todos los días en un porcentaje muy alto. Todo esto muestra que es factible utilizar esta aplicación para el aprendizaje.

Una recomendación para diseñar la aplicación es seguir el ejemplo de Zhou et al. (2018), en donde una aplicación que desarrollaron para regular la cantidad de ejercicio que una persona hace al día, tenía una particularidad que no tenían las demás pues era motivadora y ayudaba a establecer metas personalizadas cada día, pero fáciles de alcanzar. Este mismo pensamiento puede emplearse en la aplicación que apoya el aprendizaje de arreglos de este estudio, ya que se le puede incluir una sección que le genere al estudiantado metas diarias que lo motiven a medir su aprendizaje y a racionarlo de manera que pueda avanzar a un ritmo pausado pero estable.

Se recomienda tomar como base lo establecido por Alberta Education (2012), en donde se propone elaborar aplicaciones personalizadas, de acuerdo con los intereses y necesidades de la comunidad estudiantil, así como la regulación del proceso enseñanza-aprendizaje. De esta manera, la aplicación podría tener diferentes opciones para aprender que lleven al mismo resultado y contenga elementos didácticos y técnicos.

Asimismo, Chen y Denoyelles (2013), recomiendan que, para mejorar la efectividad de las aplicaciones móviles con propósitos académicos, es necesario que tanto la comunidad estudiantil como el personal docente se involucren en la comprensión del contenido y se despejen las dudas que surjan en el momento.

\section{REFERENCIAS}

Alberta Education. (2012). Programs of study. Recuperado de https://education.alberta.ca/programs-of-study/ programs-of-study/?searchMode $=3$

Arain, A., Hussain, Z., Rizvi, W., \& Vighio, M. (2017). An analysis of the influence of a mobile learning aplicaciónlication on the learning outcomes of higher education students. Universal Access in the Information Society, 17(2), 325-334.

Ayub, A., Zaini, S., Luan, W., \& Jaafar, W. (2017). The Influence of Mobile Self-efficacy, Personal Innovativeness and Readiness towards Students' Attitudes towards the use of Mobile Aplicaciones in Learning and Teaching. International Journal of Academic Research in Business and Social Sciences, 7(14), 364-374. DOI: 10.6007/ IJARBSS/v7-i14/3673

Castaño, C., \& Cabero, J. (Coords). (2013). Enseñar y aprender en entornos M-Learning. Madrid: Editorial Síntesis.

Chen, B., \& Denoyelles, A. (2013). Exploring students' mobile learning practices in higher education. Educause Review, 7. Recuperado de https://er.educause.edu/ articles/2013/10/exploring-students-mobile-learning-practices-in-higher-education

Creative Research Systems. (2006). The Survey System del Creative Research Systems. California, U.S.A. Recuperado de http://www.surveysoftware.net/sscalce.htm

De Witt, C., \& Gloerfeld, C. (2017). Mobile Learning and Higher Education. In D. Kergel, B. Heidkamp, P. Telléus, T. Rachwal, \& S. Nowakowski (Eds.), The Digital Turn in Higher Education (pp. 61-79). Wiesbaden, Germany: Springer VS.

Figueras-Maz, M., Ferrés, J., \& Mateus, J. (2018). Percepción de los/as coordinadores/as de la innovación docente en las universidades españolas sobre el uso de dispositivos móviles en el aula. Revista Prisma Social, 20, 160-179.

García, L., Ruiz, M., \& Domínguez, D. (2007). De la educación a distancia a la educación virtual ( $1^{\mathrm{a}}$ ed.). Barcelona, España: Ariel.

Hernández, R., Fernández, C., \& Baptista, P. (2006). Metodología de la investigación ( $4^{\text {a }}$ ed.). México: McGraw-Hill Interamericana.

Palmero, F. (2011). Metodología de solución de problemas. Recuperado de http://aprendizajealgoritmos.blogspot. com/2011/02/metodologia-de-solucion-de-problemas_16.html

Pechenkina, E. (2017). Developing a typology of mobile aplicaciones in higher education: A national case-study. 
Australasian Journal of Educational Technology, 33(4), 134-146.

Pechenkina, E., Laurence, D., Oates, G., Eldridge, D., \& Hunter, D. (2017). Using a gamified mobile aplicación to increase student engagement, retention and academic achievement. International Journal of Educational Technology in Higher Education, 14(31), 1-12. DOI: 10.1186/ s41239-017-0069-7

Salas, I. (2016). Modelo de enseñanza y formación en línea. En A. C. Umaña Mata, I. Salas Campos, \& V. Berrocal Carvajal (Eds.), Consideraciones para el diseño y oferta de asignaturas en línea (25-33). San José, Costa Rica: UNED.

SUTEL. (2018). Mapas de calidad SUTEL. Recuperado de https:// mapas.sutel.go.cr/
UNED. (2012). Plan de desarrollo académico 2012-2017. Recuperado de https://www.uned.ac.cr/academica/ plan_academico/insumos/PlanDesAcad_UNED_fin al.pdf

UNESCO (s.f.). El aprendizaje móvil. Recuperado de http://www. unesco.org/new/es/unesco/themes/icts/m4ed/

Vázquez, E., \& Sevillano, M. (2018). Sustainable Mobility in Higher Education Through Virtual Attendance. In U. Azeiteiro, W. Leal Filho, \& L. Aires (Eds.), Climate Literacy and Innovations in Climate Change Education (pp. 341-357). Cham: Springer. DOI: 10.1007/978-3-319-70199-8_20

Zhou, M., Mintz, Y., Fukuoka, Y., Goldberg, K., Flowers, E., Kaminsky, P., ... Aswani, A. (2018). Personalizing Mobile Fitness Aplicaciones using Reinforcement Learning. Reecuperado de http://ceur-ws.org/Vol-2068/humanize7.pdf 